\title{
Incremental value of rare genetic variants for the prediction of multifactorial diseases
}

\author{
Raluca Mihaescu' ${ }^{1}$, Michael J Pencina ${ }^{2,3}$, Alvaro Alonso ${ }^{4}$, Kathryn L Lunetta ${ }^{2,5}$, Susan R Heckbert ${ }^{6}$, \\ Emelia J Benjamin ${ }^{5,7,8}$ and A Cecile JW Janssens ${ }^{1,9^{*}}$
}

\begin{abstract}
Background: It is often assumed that rare genetic variants will improve available risk prediction scores. We aimed to estimate the added predictive ability of rare variants for risk prediction of common diseases in hypothetical scenarios.

Methods: In simulated data, we constructed risk models with an area under the ROC curve (AUC) ranging between 0.50 and 0.95 , to which we added a single variant representing the cumulative frequency and effect (odds ratio, OR) of multiple rare variants. The frequency of the rare variant ranged between 0.0001 and 0.01 and the OR between 2 and 10. We assessed the resulting $A \cup C$, increment in $A \cup C$, integrated discrimination improvement (IDI), net reclassification improvement (NRI $(>0.01)$ ) and categorical NRI. The analyses were illustrated by a simulation of atrial fibrillation risk prediction based on a published clinical risk model.
\end{abstract}

Results: We observed minimal improvement in AUC with the addition of rare variants. All measures increased with the frequency and OR of the variant, but maximum increment in AUC remained below 0.05. Increment in AUC and $\mathrm{NRI}(>0.01)$ decreased with higher AUC of the baseline model, whereas IDI remained constant. In the atrial fibrillation example, the maximum increment in AUC was 0.02 for a variant with frequency $=0.01$ and $O R=10$. IDI and NRI showed at most minimal increase for variants with frequency greater than or equal to 0.005 and OR greater than or equal to 5 .

Conclusions: Since rare variants are present in only a minority of affected individuals, their predictive ability is generally low at the population level. To improve the predictive ability of clinical risk models for complex diseases, genetic variants must be common and have substantial effect on disease risk.

\section{Background}

Genome-wide association studies (GWASs) have uncovered an incredible number of common susceptibility variants, but they explain only a small part of the heritability of complex diseases [1]. In search for the missing heritability, genetic research is investigating common variants with weak effects on disease risk, gene-gene interactions, structural variations and rare variants [2]. With the introduction of next generation sequencing, much effort is currently directed towards rare variants. Expected to have a predominant effect on protein structure, rare variants are more likely to be functional and to display strong effects on disease risk [3-6]. Sequencing of coding regions of the genome already has proved successful in

\footnotetext{
* Correspondence: cecile.janssens@emory.edu

'Department of Epidemiology, Erasmus University Medical Center,

Dr. Molewaterplein 50, Rotterdam, 3000 CA, The Netherlands

Full list of author information is available at the end of the article
}

identifying rare polymorphisms associated with common traits and complex diseases [7-10].

The predictive ability of rare variants and their potential to improve clinical risk models are uncertain for the population at large, as they are present in only a minority of the affected individuals. The predictive ability of rare variants in common diseases is understudied. Two methodological papers investigated the increment in area under the receiver operating characteristic curve (AUC) when rare variants were added to models based on common variants using simulated data $[11,12]$. They showed that the maximum increment in AUC was 0.06, but they did not provide the effect sizes of the rare variants, which makes it difficult to interpret the significance of their results. Additionally, AUC is considered an insensitive measure to detect potentially clinically important improvement in prediction [13-15]. Two new metrics were developed and rapidly gained popularity: the integrated discrimination

\section{Biomed Central}


improvement (IDI) and the net reclassification improvement (NRI) [16]. These metrics may be able to detect clinically significant improvement in prediction due to rare variants that the AUC fails to uncover.

We investigated the value of rare genetic variants for risk prediction of complex diseases. We examined the relation between the predictive ability of rare variants and their frequency, strength of effect (OR), and the predictive ability of the baseline risk model. We assessed the improvement in model performance by delta AUC ( $\triangle \mathrm{AUC})$, IDI, and NRI. To this end, we simulated a large dataset and constructed risk models based on common variants for increasing values of the baseline AUC. In separate scenarios, we added rare genetic variants with varying odds ratios (OR) and frequencies. We further used hypothetical data that replicated the empirical populations used to derive a recently published clinical model for atrial fibrillation (AF) [17]. This common cardiac arrhythmia is associated with increased morbidity, mortality, and significant healthcare costs [18]. Numerous common genetic variants associated with atrial fibrillation risk have been identified [19-22] and rare genetic variants are expected to improve the detection of at-risk individuals [23,24].

\section{Methods}

\section{Simulation of data}

First, we used a simulation procedure to investigate the effect of the predictive accuracy of the baseline model on the discrimination of the model updated with rare variants. The modeling procedure has been described in detail by Janssens et al. [25]. In short, this procedure creates a dataset of genotypes for a hypothetical population. Genotypes, coded as 0,1 , or 2 based on the number of risk alleles, are assigned in such a way that the allele frequencies of the genetic variants match specified values and are in Hardy-Weinberg equilibrium. By changing the number, frequency, and ORs of simulated variants we created baseline models with an AUC ranging between 0.50 and 0.95 . We added rare genetic variants to the simulated dataset of common variants. Rare variants were simulated as a single variant representing multiple rarer variants. That is, for example, 20 independent rare variants each with a frequency of 1 in 2,000 individuals can collectively be viewed as a single variant with a frequency of 0.01 . The variant was coded as 1 or 0 if the individual carried any or none of the risk alleles. We simulated rare variants with a frequency of 0.0001 , $0.001,0.005$, or 0.01 , and an OR of 2,5 , or 10 . We used arbitrary values for the parameters of the rare genes, but based our choice on the literature [3,26-28].

To compare the added value of rare and common variants for risk prediction, we also simulated 10 to 100 common variants each with a risk allele frequency of
0.05 or 0.30 and an OR of 1.10 or 1.05 . We have used these parameters because most of the approximately 400 single nucleotide polymorphisms (SNPs) discovered in 100 recent GWAS had an OR of approximately 1.10 and future GWAS efforts are expected to uncover SNPs with even lower effect sizes [28]. Disease risk was $4 \%$ as in the AF example, or $10 \%$ to examine the impact of higher disease risks on the measures of predictive ability. In the simulations, disease risk can be interpreted as a disease incidence, for example, a disease incidence over 5 years. For both the main simulations and the AF example, population size was 200,000 for scenarios in which rare variants were added and 20,000 for scenarios in which common variants were added. Predicted risks for each individual were obtained from logistic regression analyses, were calculated in the range 0 to 1 and were rounded to two decimal points.

\section{Simulation study of atrial fibrillation Background for choosing the example of atrial fibrillation} Complex diseases can be multifactorial, that is, caused by an intricate effect of multiple environmental and genetic risk factors, but can also include monogenic forms. One such example is AF, which consist of a rare familial form that is a monogenetic disease and a common non-familial form [29]. Targeted use of prevention strategies is warranted to reduce the burden of AF, which requires accurate detection of individuals at high risk. Algorithms for detection of individuals at risk, based on routinely collected clinical risk factors, have already been developed and validated in various populations $[17,30,31]$. The predictive accuracy of these clinical scores leaves ample opportunity for improvement, and so fuels the research for finding new biomarkers, including genetic variants $[23,32,33]$. Several susceptibility variants for AF have been found [19-21] but their combined predictive ability is low as they explain only a fraction of the heritability [1]. While sequencing efforts are ongoing for AF, research focused on the potential use of rare variants for risk prediction of AF becomes very relevant $[23,24]$. We assessed the incremental value of rare genetic variants over an existing clinical risk score for AF.

\section{Methods for constructing the dataset of clinical and genetic risk factors}

To assess the value of rare variants for AF risk prediction we simulated a hypothetical population that reflected the characteristics of the community-based cohort in which the clinical risk score was developed (that is, the combination of Atherosclerosis Risk in Communities Study, Cardiovascular Health Study and Framingham Heart Study; please see Additional file for details on study design) [34-36]. We simulated the distribution of clinical and genetic risk factors separately in individuals with and 
without the outcome by random sampling from a multivariate normal distribution. To derive categorical clinical variables and genetic variants, we transformed the corresponding continuous variables into categorical variables. We simulated clinical variables to be correlated, as observed in the empirical population (see Additional file, Table S3). We assumed that genetic variants were uncorrelated with one another and were uncorrelated with clinical risk variables. Detailed information about the simulation strategy is provided in the Supplementary Methods (see Additional file).

\section{Description of the clinical model}

Variables included in the clinical risk score were: age, race, smoking status, weight, height, systolic blood pressure (SBP), diastolic blood pressure (DBP), diabetes, medication for hypertension, history of congestive heart failure, and history of myocardial infarction (see Additional file, Table S1 for the distribution of clinical variables). In the empirical dataset, that is, the combination of Atherosclerosis Risk in Communities Study, Cardiovascular Health Study and Framingham Heart Study, the outcome was defined as AF during 5 years of follow-up. Individuals were free of AF at the beginning of followup. The disease incidence was $4 \%$. Simulations accurately replicated the empirical data (see Additional file, Table S4).

\section{Description of genetic variables}

We used the same parameters for the rare variants as in the simulation scenarios where we varied the baseline AUC. To estimate the added value of recently identified susceptibility single nucleotide polymorphisms (SNPs) for $\mathrm{AF}$, we added to the clinical variables 10 genetic variants with the same frequency and OR as the top 10 (that is, in terms of $P$ value) uncorrelated SNPs from a recent meta-analysis performed in the CHARGE AF consortium [22] (see Additional file, Table S2).

\section{Metrics}

We assessed discrimination of the baseline, genetic, and combined models; improvement in discrimination; and clinical usefulness of updating the baseline model with genetic variants. We used AUC as a global measure of discrimination. AUC indicates the degree to which the predicted risks can discriminate between individuals who will and will not develop the disease. AUC generally ranges from 0.50 (equal to tossing a coin) to 1.00 (perfect discrimination). We used the increment in AUC ( $\triangle \mathrm{AUC}), \mathrm{IDI}$, and continuous NRI as measures of global improvement in discrimination. IDI was calculated as the difference in mean predicted probabilities between cases and controls between the two models [16]. NRI is an overall measure of correct reclassification of cases to higher risk categories and of controls to lower risk categories [16]. The continuous NRI $[\mathrm{NRI}(>0)]$ does not use categories but takes into account any increase or decrease in predicted risk produced by the model update [37]. Since we rounded risks to 0.01 (that is, 1\%) the NRI without categories used here is denoted as NRI $(>0.01)$. In other words the minimal change in risk is 0.01 . We used categorical NRI to assess clinical usefulness. Clinical usefulness concerns the reclassification of individuals in risk categories that leads to changes in preventive or therapeutic interventions. We defined three risk categories by using the risk cutoffs of $2.5 \%$ and $5 \%$, similar to those used the evaluation of the clinical risk score for AF [17]. We also report the NRI in cases and controls separately, as this may provide additional insight into the impact of model update [16,37]. For scenarios with various baseline AUC, we calculated $\triangle \mathrm{AUC}, \mathrm{NRI}(>0.01)$, and IDI. For scenarios with the clinical risk score we calculated $\triangle \mathrm{AUC}$, IDI, $\mathrm{NRI}(>0.01)$, and categorical NRI.

Reported measures are median results from 200 simulations unless stated otherwise. All analyses were performed using the $\mathrm{R}$ programming language, version 2.11.1 [38].

\section{Results}

\section{Simulation analyses}

Figure 1 shows that, for a disease risk of $4 \%$, the median AUC and NRI $(>0.01)$ only improved when variants were not very rare and had higher ORs, and only when baseline AUC values were in the lower range. Across higher baseline AUC values, the median NRI(>0.01) became negative, suggesting that rare variants produced more wrong than correct risk reclassifications. The median IDI was close to zero for very rare variants and minimally increased with higher frequency and OR of the rare variants. The median IDI was constant across most baseline AUC values. When disease risk was higher (that is, 10\%), most performance measures slightly increased compared to the scenarios with lower disease risk (see Additional file, Figure S1). For rare variants with $\mathrm{OR}=10$ and frequency $\geq 0.005$, the median increment in AUC varied between 0.01 and 0.05 depending on the value of the baseline AUC. The median NRI (>0.01) varied between 0.18 and 0.55 and, in contrast to the scenario with the lower disease risk, increased with a higher baseline AUC.

As a comparison, we investigated the addition of 10 to 100 common variants, each with a frequency of 0.05 and an OR of 1.10 or a frequency of 0.30 and an OR of 1.05. We found a higher increase in AUC compared to the addition of rare variants (see Figure 2). NRI $(>0.01)$ was mostly positive and increased with the number of variants added. In contrast, IDI was minimal even with the addition of 100 variants. Surprisingly, although the increment in AUC was higher, the IDI was in some 


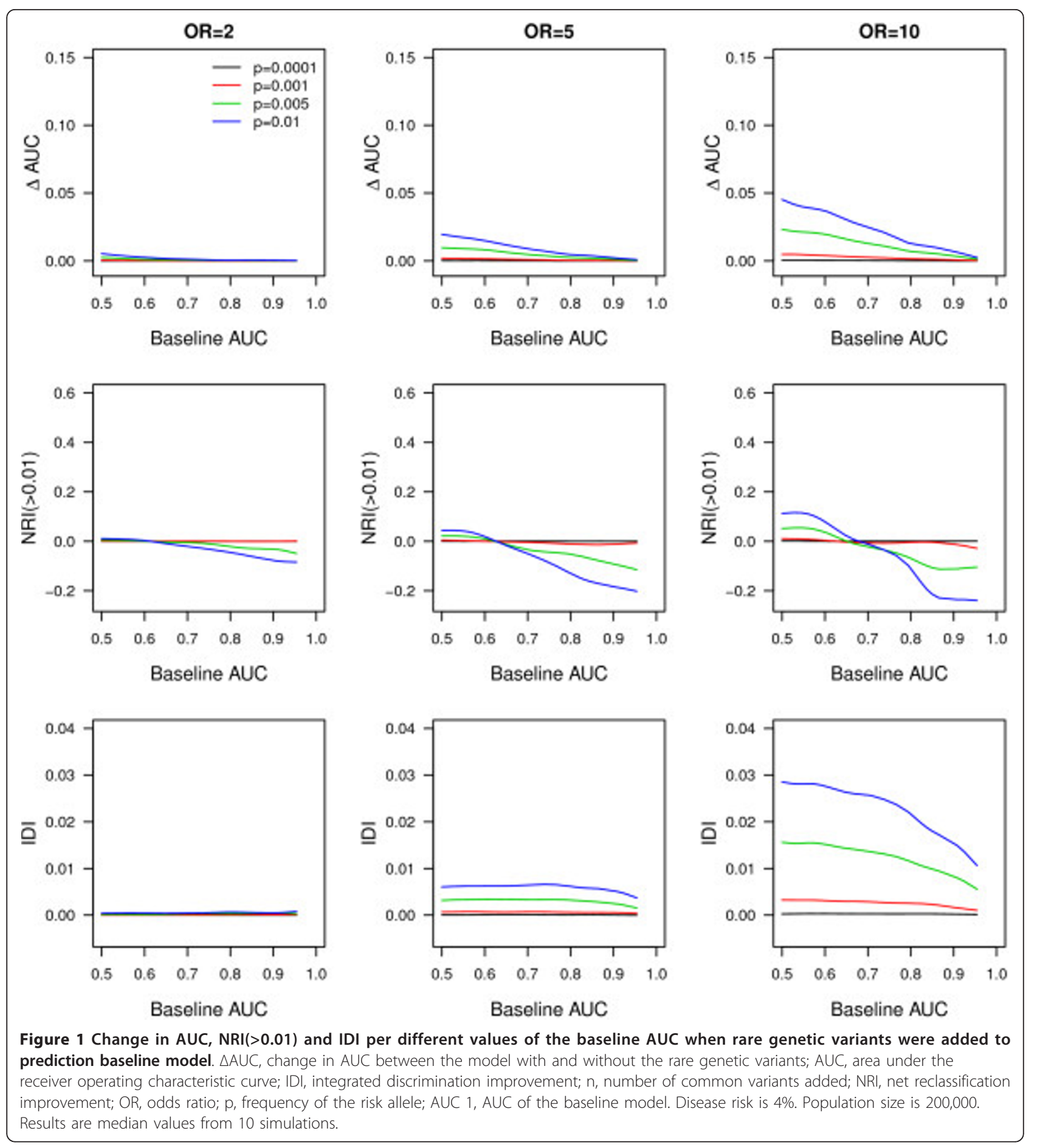

instances lower for common variants compared to rare variants. As such, across low baseline AUC values rare variants with $\mathrm{OR}=10$ and frequency $\geq 0.005$ showed higher IDI than 100 common variants. This trend was seen also when disease risk was higher (that is, 10\%) (see Additional file, Figure S2). Furthermore, rare variants with $\mathrm{OR}=10$ and frequency $\geq 0.005$ showed also higher NRI(>0.01) across higher baseline AUC values compared to 100 common variants (see Additional file, Figure S2).

To investigate the added value of rare and common variants at the individual level, we additionally assessed the magnitude of change in absolute risk at reclassification. Having a rare variant substantially increased the 

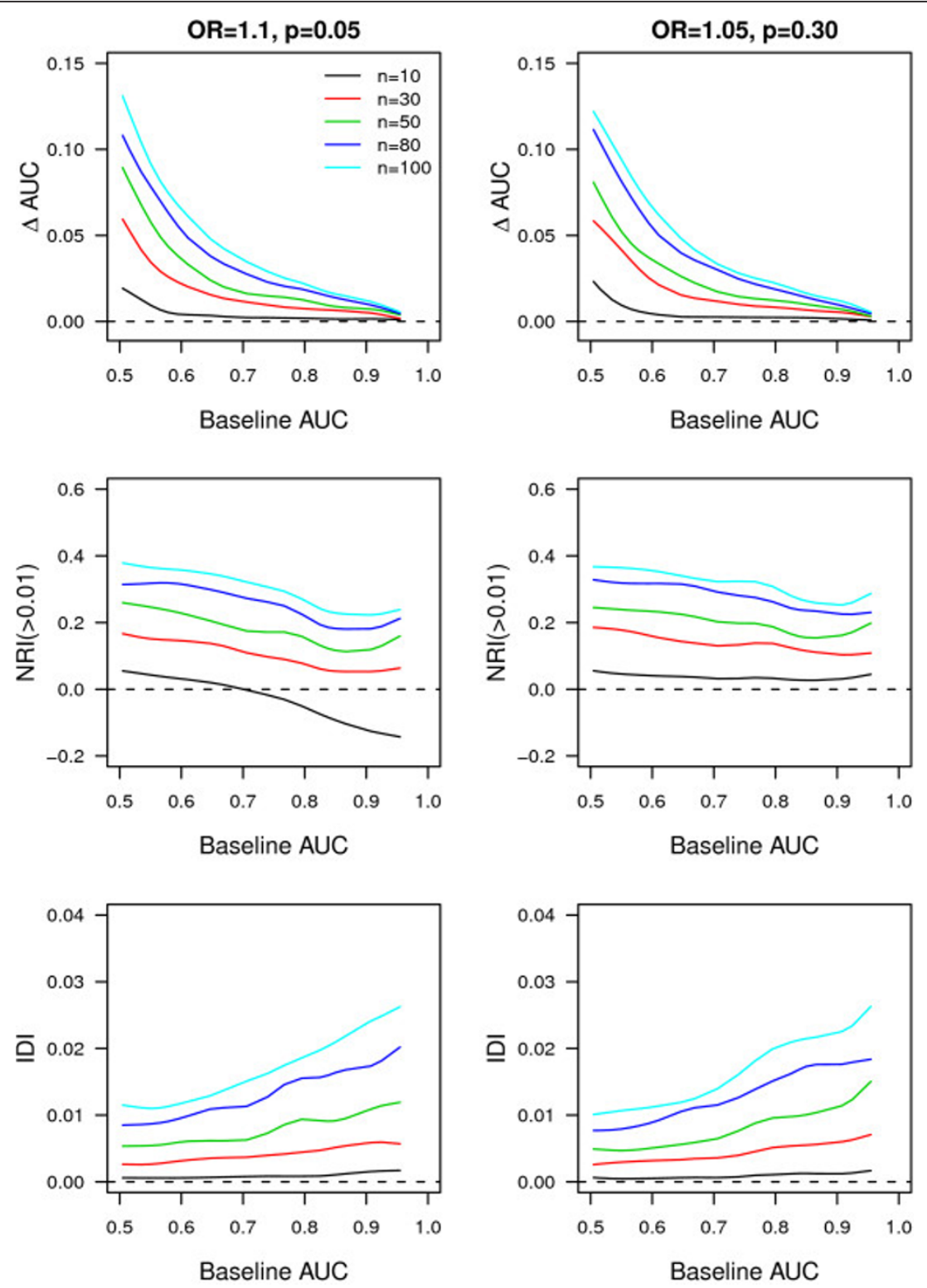

Figure 2 Change in AUC, $\mathrm{NRI}(>0.01)$ and IDI per different values of the baseline AUC when common genetic variants were added to prediction baseline model. $\triangle A \cup C$, change in AUC between the model with and without the common genetic variants; AUC, area under the receiver operating characteristic curve; IDI, integrated discrimination improvement; $n$, number of common variants added; NRI, net reclassification improvement; OR, odds ratio; p, frequency of the risk allele; AUC 1, AUC of the baseline model. Disease risk is 4\%. Population size is 20,000. Results are median values from 10 simulations.

risk in $<1 \%$ of both cases and controls when disease risk was $4 \%$ (median increase in absolute risk: 0.35 in cases and 0.24 in controls; see Figure 3a). When disease risk was $10 \%$ the risk in $10 \%$ of cases largely increased while the risk in $<1 \%$ of controls increased only marginally (median increase in absolute risk: 0.78 in cases and 0.02 in controls; see Figure $3 b$ ). The median decrease was negligible in both cases and controls that did not carry the risk variant (that is, -0.01). In contrast, when 100 common variants were added to the model and the disease risk was $4 \%$, the risk minimally increased or decreased in individuals that were reclassified to higher or lower risk categories (median increase in absolute risk: 0.03 in cases and 0.02 in controls, median decrease: -0.02 and -0.01 ; see Figure 3c). When common variants were added, about a half of the cases and controls moved in the right direction while around one-quarter moved in the wrong direction. Similar results were observed when disease risk was 10\% (see Figure 3d). Besides the individuals that carried the risk variant, an 

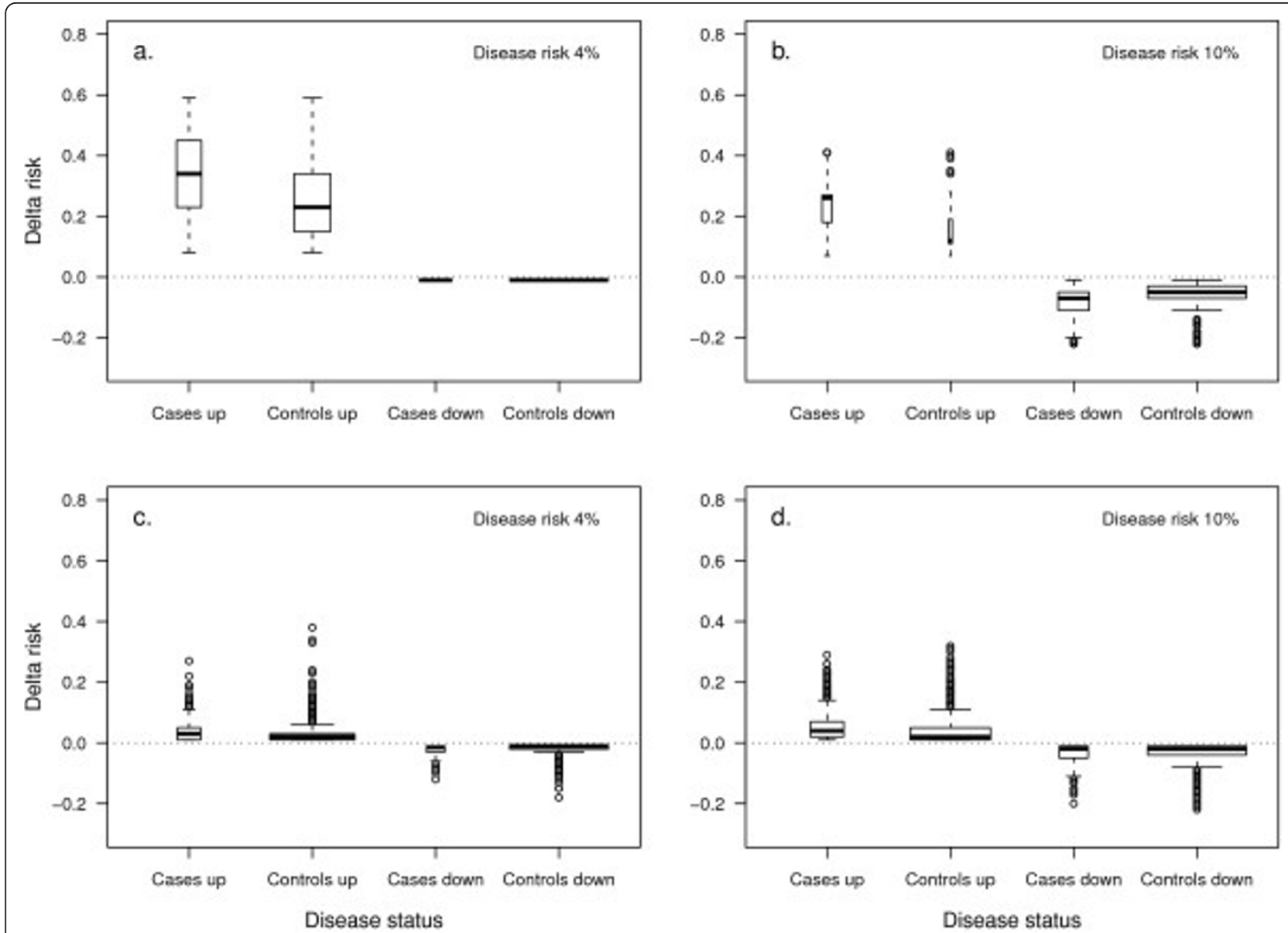

Figure 3 Change in absolute risk at model update with rare and common genetic variants. On the $\times$ axis is shown the correct reclassification of cases and controls (that is, Cases up; Controls down) and incorrect reclassification (that is, Cases down; Controls up) when rare variants with a cumulative OR of 10 and frequency of 0.01 (Figures 3a, b) or 100 common variants each with a OR of 1.05 and a frequency of 0.30 (Figures $3 c, d$ ) are added to a baseline model with an $A \cup C=0.70$. The bold line shows the median, the boxes indicate the interquartile ranges (range, 25-75\%), and the whiskers present 1.5 times the interquartile range. Box widths are proportional to the square-root of the number of individuals in the groups. Disease risk is $4 \%$ in Figures $3 \mathrm{a}$ and $\mathrm{c}$, and $10 \%$ in Figures $3 \mathrm{~b}$ and $\mathrm{d}$. The plot is obtained from one simulation using 200,000 individuals for Figures $3 a$ and b, and 20,000 individuals for Figures $3 c$ and d.

increase in risk was observed also in some individuals that did not carry a rare variant. This risk increase was minimal and was due to a difference in beta coefficients between the two regression models (data not shown).

\section{Clinical example: genetic prediction of atrial fibrillation}

The median AUC of the clinical model was 0.76 (95\% confidence interval, 0.75 to 0.78 ). Rare variants improved the AUC of the clinical model only when they were more frequent and had very high OR (see Table 1). Adding a rare variant with a frequency of 0.01 increased AUC by 0.02 when OR was 10, but did not improve AUC when OR was 2. IDI and NRI were zero for very rare variants, that is, when frequency was 0.0001 or 0.001 . IDI minimally increased with a higher frequency and OR of the rare variant (see Table 1). A variant with frequency of 0.01 and an OR of 10 yielded an IDI of 0.03 . NRI $(>0.01)$ was negative in most scenarios. The higher the frequency and OR of the rare variant, the larger the negative value of $\mathrm{NRI}(>0.01)$. In contrast to the $\mathrm{NRI}(>0.01)$, categorical NRI and NRI in controls, but not in cases, were positive and minimally increased with the frequency and OR of the rare variants. Adding 10 variants with empirical ORs and frequencies showed a minimal improvement in all model performance measures.

\section{Discussion}

Using a hypothetical population, we have shown that rare variants only minimally improved AUC and did not yield clinically relevant positive $\mathrm{NRI}(>0.01)$ and IDI when disease risk was low. Rare variants produced larger increments in AUC when the baseline model had lower AUC, but in these scenarios $\mathrm{NRI}(>0.01)$ and IDI remained close to zero. Addition of rare variants to the 
Table 1 Performance of genetic and combined (clinical and genetic) risk models for AF using rare and common variants.

\begin{tabular}{|c|c|c|c|c|c|c|c|c|c|c|}
\hline \multirow[t]{2}{*}{ OR } & \multirow[t]{2}{*}{ Frequency } & \multirow[t]{2}{*}{ Variants $(n)$} & \multicolumn{3}{|l|}{ AUC } & \multirow[t]{2}{*}{ IDI } & \multirow[t]{2}{*}{$\mathrm{NRI}(>0.01)$} & \multicolumn{3}{|c|}{ NRI categorical } \\
\hline & & & Genetic & Combined & $\Delta$ & & & Total & Cases & Controls \\
\hline \multicolumn{11}{|c|}{ Rare variants } \\
\hline \multirow[t]{4}{*}{2} & 0.0001 & 1 & 0.50 & 0.76 & 0 & 0 & 0 & 0 & 0 & 0 \\
\hline & 0.001 & 1 & 0.50 & 0.76 & 0 & 0 & 0 & 0 & 0 & 0 \\
\hline & 0.005 & 1 & 0.50 & 0.76 & 0 & 0 & -0.01 & 0 & 0 & 0 \\
\hline & 0.01 & 1 & 0.51 & 0.76 & 0 & 0 & -0.03 & 0 & 0 & 0 \\
\hline \multirow[t]{4}{*}{5} & 0.0001 & 1 & 0.50 & 0.76 & 0 & 0 & 0 & 0 & 0 & 0 \\
\hline & 0.001 & 1 & 0.50 & 0.76 & 0 & 0 & -0.01 & 0 & 0 & 0 \\
\hline & 0.005 & 1 & 0.51 & 0.76 & 0 & 0 & -0.05 & 0.01 & 0.01 & 0 \\
\hline & 0.01 & 1 & 0.52 & 0.77 & 0.01 & 0.01 & -0.10 & 0.02 & -0.01 & 0.03 \\
\hline \multirow[t]{4}{*}{10} & 0.0001 & 1 & 0.50 & 0.76 & 0 & 0 & 0 & 0 & 0 & 0 \\
\hline & 0.001 & 1 & 0.50 & 0.76 & 0 & 0 & -0.03 & 0.00 & 0 & 0.01 \\
\hline & 0.005 & 1 & 0.52 & 0.77 & 0.01 & 0.02 & -0.11 & 0.02 & -0.01 & 0.03 \\
\hline & 0.01 & 1 & 0.54 & 0.78 & 0.02 & 0.03 & -0.13 & 0.04 & -0.02 & 0.06 \\
\hline \multicolumn{11}{|c|}{ Common variants } \\
\hline $1.14-1.45^{\mathrm{a}}$ & $0.03-0.84^{*}$ & 10 & 0.59 & 0.77 & 0.01 & 0.01 & 0.20 & 0.04 & 0.01 & 0.04 \\
\hline
\end{tabular}

a Using parameters from the top 10 (that is, in terms of $P$ value) uncorrelated SNPs in the CHARGE AF meta-analysis; in the table are listed the range of OR and allele frequency [22]. Variables included in the clinical risk score were: age, weight, height, systolic blood pressure (SBP), diastolic blood pressure (DBP), diabetes, medication for hypertension, history of congestive heart failure, history of myocardial infarction, smoking status, and race. Disease risk is $4 \%$ and population size is 200,000 for rare variants scenarios and 20,000 for common variants scenarios. Results are median values from 200 simulations.

$A \cup C$, area under the receiver operating characteristic curve; $\triangle A \cup C$, change in AUC between the model with and without genetic variants; IDI, integrated discrimination improvement; NRI, net reclassification improvement (cutoffs 2.5\% and 5\%); OR, odds ratio.

baseline model largely increased predicted risks for the few individuals carrying the risk variant, whereas predicted risks were only slightly decreased for those who did not carry the variant. For a higher disease risk, rare variants with strong effects showed improvement in AUC across a wider range of baseline AUC values and significant positive $\mathrm{NRI}(>0.01)$ and IDI. Addition of rare variants to the baseline model largely increased predicted risks only in cases, as expected.

Before addressing the implications of these results for future research, we discuss several methodological aspects of our study that might have impacted the results. First, we modeled rare variants as a single variant. This is a common procedure used to investigate the association of multiple extremely rare variants with disease risk and does not affect the results presented here [9,39]. Second, we assumed that each genetic variant was uncorrelated with other variants and clinical risk factors. While linkage equilibrium between rare variants is a very realistic assumption, rare variants may be in linkage disequilibrium with common variants. In fact, it has been suggested that common variants may share a haplotype with the true rare causal variants [6]. Third, genetic variants may be associated with intermediate risk factors for disease, which are often the variables included in traditional clinical risk scores. Such correlations would likely decrease the impact of the variants and hence show less improvement in performance than reported in this paper.
We have shown that, from a population perspective, rare variants are only useful for risk prediction of complex diseases when they have strong effects on disease risk, when they are not too rare and when the risk of disease is high. Figure 1 shows that when disease risk was $4 \%$ the addition of rare variants resulted in an improvement in AUC only when the baseline AUC was low. As shown, this trend was more pronounced when rare variants had higher OR. NRI $(>0.01)$ showed a minimal added value of rare variants only when baseline AUC was lower $(\leq 0.70)$ and variants had very strong effects $(\mathrm{OR}=10)$. When baseline AUC was $\geq 0.80$, the $\mathrm{NRI}(>0.01)$ indicated that overall more wrong reclassifications of risk were done by addition of rare variants. To summarize, rare variants only improved discrimination when baseline AUC values were low, but even then the improvement was minimal. Only when the $\mathrm{OR}$ of the rare variant was very large, its frequency higher and disease risk high, were the AUC and NRI $(>0.01)$ improved across a wider range of baseline AUC. If the expected effect sizes might be smaller than previously thought, the predictive ability of rare variants will be even lower than our results indicate. The NRI $(>0.01)$ and IDI values were higher than those observed with the addition of 100 common variants with a frequency and OR as used in this study. Thus, despite a lower improvement in AUC, rare variants may result in larger improvements in NRI $(>0.01)$ and IDI compared to common variants. This apparent discrepancy in observations is explained by the 
fact that AUC only considers the rank in predicted risks, not actual values, whereas NRI and IDI do depend on actual magnitude of changes in predicted risks before and after updating the model. Rare variants with strong effect by definition have a substantial impact on disease risk, albeit for a small group of individuals.

Interestingly, the degree of precision had a large impact on the global improvement of discrimination as measured by continuous NRI. The discrepancy in results was most striking for the rare variants. As such, we observed a NRI $(>0)$ of 0.17 when a rare variant with OR of 10 and frequency of 0.01 was added to the clinical AF model and predicted risks were not rounded, compared to an NRI $(>0.01)$ of -0.13 when risks were rounded to two decimal points. This is likely explained by the fact that, by definition, most individuals did not carry the rare risk variant and this resulted in a very small decrease in risk for most individuals, a change that was not captured when risks were rounded. In contrast, the difference in AUC and categorical NRI between non-rounded and rounded risks was minimal, that is, at most 0.01 in a few scenarios from the AF example. This raises the question what is the amount of precision to be reported for risk predictions and what is the most appropriate continuous NRI measure.

\section{Conclusions}

In conclusion, we have shown that addition of rare variants to baseline risk models that include clinical or genetic risk factors resulted in model improvement only when the rare variants had strong effects on disease risk. This improvement was larger with a higher disease risk because the odds ratios lead to different likelihood ratios when the disease is more common. We have also shown that rare variants largely increased the risk in some individuals, while most individuals were reclassified to a slightly decreased risk. Very rare variants, by definition, occur in only few individuals that ultimately develop the disease and therefore have poor sensitivity and a limited predictive ability. This means that most individuals will either not be reclassified into another risk category or will be reclassified on the basis of clinically irrelevant changes in predicted risks. Counterintuitively, most individuals who will develop the disease will see their risk slightly decreased after testing for rare variants. Although this decrease in disease risk is minimal, individuals with predicted risks just above the threshold may be moved to a lower risk category. In the case of AF, a disease associated with stroke and increased mortality [40], this would mean that many individuals would not benefit from the potentially lifesaving preventive measures.

While rare variants are unlikely to improve the prediction of common diseases in the population, they do have substantial impact on disease risk in carriers of the rare variants. When rare variants have very strong effects on disease risk, they are probably more aggregated within certain families and resemble a Mendelian transmission. It would be of high interest to compare family history information [41] with tests including rare variants and, further, to investigate if such variants can be more predictive in families with positive family history. Apart from such exceptions, it should be anticipated that the study of rare variants will have its largest contribution in advancing our understanding of disease pathophysiology.

\section{Description of additional data files}

The following additional data are available with the online version of this paper. The Additional file includes information on study design and baseline characteristics in Atherosclerosis Risk in Communities Study, Cardiovascular Health Study and Framingham Heart Study and describes the top 10 independent SNPs, change in AUC, $\mathrm{NRI}(>0.01)$, and IDI per different values of the baseline AUC when rare or common genetic variants are added to prediction baseline model and additional information on the methods.

\section{List of abbreviations}

AF: atrial fibrillation; AUC: area under the ROC curve; $\triangle A U C$ : increment in AUC; DBP: diastolic blood pressure; GWAS: genome-wide association study; IDI: integrated discrimination improvement; NRI: net reclassification improvement; OR: odds ratio; SBP: systolic blood pressure; SNPs: single nucleotide polymorphisms.

\section{Competing interests}

The authors declare that they have no competing interests.

\section{Authors' contributions}

RM, MJP, and ACJWJ conceived the study and drafted the manuscript. RM performed the statistical analysis. AA, KLL, SRH, and EJB participated in the design and helped to draft the manuscript. All authors read and approved the final manuscript.

\section{Acknowledgements}

This work was supported by the Netherlands Organization for Scientific Research (Vidi grant to ACJWJ); Centre for Medical Systems Biology in the framework of the Netherlands Genomics Initiative (to ACJWJ); Erasmus Trustfonds (to RM); National Institutes of Health/American Recovery and Reinvestment Act Risk Prediction of Atrial Fibrillation (1RC1HL101056 to MP and EJB); National Institutes of Health/National Heart, Lung, and Blood Institute (RC1HL099452 to AA; HL068986 to SRH; HL092577 to EJB, KLL, and SRH; HL080295 to SRH; 1R01HL102214 to EJB; N01-HC 25195 to EJB); and the American Heart Association (09SDG2280087 to AA).

\section{Authors' details}

${ }^{1}$ Department of Epidemiology, Erasmus University Medical Center, Dr. Molewaterplein 50, Rotterdam, 3000 CA, The Netherlands. ${ }^{2}$ Department of Biostatistics, Boston University, 801 Massachusetts Avenue, Boston, MA 02118, USA. ${ }^{3}$ Harvard Clinical Research Institute, 930-W Commonwealth Avenue, Boston, MA 02215-1212, USA. ${ }^{4}$ Division of Epidemiology and Community Health, School of Public Health, University of Minnesota, $1300 \mathrm{~S}$. Second Street, Minneapolis, MN 55454-1015, USA. ${ }^{5}$ The National Heart, Lung, and Blood Institute's Framingham Heart Study, 73 Mt. Wayte Avenue, Framingham, MA 01702-5827, USA. 'Department of Epidemiology, University of Washington, Seattle, 1959 NE Pacific Street, Seattle, WA 98195-7236, USA. ${ }^{7}$ Cardiology and Preventive Medicine Section, Boston University School of Medicine, Boston, 715 Albany Street, MA 02118, USA. ${ }^{8}$ Department of 
Epidemiology, Boston University School of Public Health, Boston, 715 Albany Street, MA 02118, USA. ' Emory University, Rollins School of Public Health, 1518 Clifton Road, Atlanta, GA 30322 USA.

Received: 11 April 2013 Revised: 3 August 2013

Accepted: 20 August 2013 Published: 20 August 2013

\section{References}

1. Jostins L, Barrett JC: Genetic risk prediction in complex disease. Hum Mol Genet 2011, 20:R182-188.

2. Makowsky R, Pajewski NM, Klimentidis YC, Vazquez Al, Duarte CW, Allison DB, de los Campos G: Beyond missing heritability: prediction of complex traits. PLoS Genet 2011, 7:e1002051.

3. Bodmer W, Bonilla C: Common and rare variants in multifactorial susceptibility to common diseases. Nat Genet 2008, 40:695-701.

4. Gorlov IP, Gorlova OY, Frazier ML, Spitz MR, Amos Cl: Evolutionary evidence of the effect of rare variants on disease etiology. Clin Genet 2011, 79:199-206.

5. Visscher PM, Goddard ME, Derks EM, Wray NR: Evidence-based psychiatric genetics, AKA the false dichotomy between common and rare variant hypotheses. Mol Psychiatry 2012, 17:474-485.

6. Wang K, Dickson SP, Stolle CA, Krantz ID, Goldstein DB, Hakonarson H: Interpretation of association signals and identification of causal variants from genome-wide association studies. Am J Hum Genet 2010, 86:730-742.

7. Yi X, Liang Y, Huerta-Sanchez E, Jin X, Cuo ZX, Pool JE, Xu X, Jiang H, Vinckenbosch N, Korneliussen TS, Zheng H, Liu T, He W, Li K, Luo R, Nie X, Wu H, Zhao M, Cao H, Zou J, Shan Y, Li S, Yang Q, Ni P, Tian G, Xu J, Liu X, Jiang $T$, Wu R, et al: Sequencing of 50 human exomes reveals adaptation to high altitude. Science 2010, 329:75-78.

8. Price AL, Kryukov GV, de Bakker PI, Purcell SM, Staples J, Wei LJ, Sunyaev SR: Pooled association tests for rare variants in exon-resequencing studies. Am J Hum Genet 2010, 86:832-838.

9. Ji W, Foo JN, O'Roak BJ, Zhao H, Larson MG, Simon DB, Newton-Cheh C, State MW, Levy D, Lifton RP: Rare independent mutations in renal salt handling genes contribute to blood pressure variation. Nat Genet 2008, 40:592-599.

10. Nejentsev S, Walker N, Riches D, Egholm M, Todd JA: Rare variants of IFIH1, a gene implicated in antiviral responses, protect against type 1 diabetes. Science 2009, 324:387-389.

11. Wei C, Lu Q: Collapsing ROC approach for risk prediction research on both common and rare variants. BMC Proceedings 2011, Suppl 9: S42.

12. Wu C, Walsh KM, ATD, JH, ZW : Disease risk prediction with rare and common variants. BMC Proceedings 2011, , Suppl 9: S61.

13. Cook NR: Use and misuse of the receiver operating characteristic curve in risk prediction. Circulation 2007, 115:928-935.

14. Pepe MS, Janes HE: Gauging the performance of SNPs, biomarkers, and clinical factors for predicting risk of breast cancer. I Natl Cancer Inst 2008, 100:978-979.

15. Pepe MS, Feng Z, Huang $Y$, Longton G, Prentice R, Thompson IM, Zheng Y: Integrating the predictiveness of a marker with its performance as a classifier. Am J Epidemiol 2008, 167:362-368.

16. Pencina MJ, D'Agostino RB Sr, D'Agostino RB Jr, Vasan RS: Evaluating the added predictive ability of a new marker: from area under the ROC curve to reclassification and beyond. Stat Med 2008, 27:157-172, discussion 207-112.

17. Alonso A, Krijthe BP, Aspelund T, Stepas KA, Pencina MJ, Moser CB, Sinner MF, Sotoodehnia N, Fontes JD, Janssens AC, Kronmal RA, Magnani JW, Witteman JC, Chamberlain AM, Lubitz SA, Schnabel RB, Agarwal SK, McManus DD, Ellinor PT, Larson MG, Burke GL, Launer L, Hofman A, Levy D, Gottdiener JS, Kaab S, Couper D, Harris TB, Soliman EZ, Stricker $\mathrm{BH}$, et al: Simple risk model predicts incidence of atrial fibrillation in a racially and geographically diverse population: the CHARGE-AF Consortium. J Am Heart Assoc 2013, 2:e000102.

18. Wolowacz SE, Samuel M, Brennan VK, Jasso-Mosqueda JG, Van Gelder IC: The cost of illness of atrial fibrillation: a systematic review of the recent literature. Europace 2011, 13:1375-1385.

19. Benjamin EJ, Rice KM, Arking DE, Pfeufer A, van Noord C, Smith AV, Schnabel RB, Bis JC, Boerwinkle E, Sinner MF, Dehghan A, Lubitz SA D'Agostino RB, Lumley T, Ehret GB, Heeringa J, Aspelund T, Newton-Cheh C, Larson MG, Marciante KD, Soliman EZ, Rivadeneira F, Wang TJ, Eiriksdottir G, Levy D, Psaty BM, Li M, Chamberlain AM, Hofman A, Vasan RS, et al:
Variants in ZFHX3 are associated with atrial fibrillation in individuals of European ancestry. Nat Genet 2009, 41:879-881.

20. Gudbjartsson DF, Arnar DO, Helgadottir A, Gretarsdottir S, Holm H, Sigurdsson A, Jonasdottir A, Baker A, Thorleifsson G, Kristjansson K, Palsson A, Blondal T, Sulem P, Backman VM, Hardarson GA, Palsdottir E, Helgason A, Sigurjonsdottir R, Sverrisson JT, Kostulas K, Ng MC, Baum L, So WY, Wong KS, Chan JC, Furie KL, Greenberg SM, Sale M, Kelly P, MacRae CA, et al: Variants conferring risk of atrial fibrillation on chromosome 4q25. Nature 2007, 448:353-357.

21. Ellinor PT, Lunetta KL, Glazer NL, Pfeufer A, Alonso A, Chung MK, Sinner MF, de Bakker PI, Mueller M, Lubitz SA, Fox E, Darbar D, Smith NL, Smith JD, Schnabel RB, Soliman EZ, Rice KM, Van Wagoner DR, Beckmann BM, van Noord C, Wang K, Ehret GB, Rotter II, Hazen SL, Steinbeck G, Smith AV, Launer $L$, Harris TB, Makino S, Nelis M, et al: Common variants in KCNN3 are associated with lone atrial fibrillation. Nat Genet 2010, 42:240-244.

22. Ellinor PT, Lunetta $\mathrm{KL}$, Albert $\mathrm{CM}$, Glazer $\mathrm{NL}$, Ritchie $\mathrm{MD}$, Smith $\mathrm{AV}$, Arking DE, Muller-Nurasyid M, Krijthe BP, Lubitz SA, Bis JC, Chung MK, Dorr M, Ozaki K, Roberts JD, Smith JG, Pfeufer A, Sinner MF, Lohman K, Ding J, Smith NL, Smith JD, Rienstra M, Rice KM, Van Wagoner DR, Magnani JW, Wakili R, Clauss S, Rotter Jl, Steinbeck G, et al: Meta-analysis identifies six new susceptibility loci for atrial fibrillation. Nat Genet 2012 44:670-675.

23. O'Donnell CJ, Nabel EG: Genomics of cardiovascular disease. N Engl J Med 2011, 365:2098-2109.

24. Parvez B, Darbar D: The "missing" link in atrial fibrillation heritability. J Electrocardiol 2011, 44:641-644.

25. Janssens AC, Aulchenko YS, Elefante S, Borsboom GJ, Steyerberg EW, van Duijn CM: Predictive testing for complex diseases using multiple genes: fact or fiction?. Genet Med 2006, 8:395-400.

26. Oexle K: A remark on rare variants. J Hum Genet 2010, 55:219-226.

27. Ionita-Laza I, Ottman R: Study designs for identification of rare disease variants in complex diseases: the utility of family-based designs. Genetics 2011, 189:1061-1068

28. Pawitan $Y$, Seng $K C$, Magnusson PK: How many genetic variants remain to be discovered? PLoS One 2009, 4:e7969

29. Tsai $C T$, Lai LP, Hwang JJ, Lin JL, Chiang FT: Molecular genetics of atrial fibrillation. J Am Coll Cardiol 2008, 52:241-250.

30. Schnabel RB, Sullivan LM, Levy D, Pencina MJ, Massaro JM, D'Agostino RB, Newton-Cheh C, Yamamoto JF, Magnani JW, Tadros TM, Kannel WB, Wang TJ, Ellinor PT, Wolf PA, Vasan RS, Benjamin EJ: Development of a risk score for atrial fibrillation (Framingham Heart Study): a communitybased cohort study. Lancet 2009, 373:739-745

31. Chamberlain AM, Agarwal SK, Folsom AR, Soliman EZ, Chambless LE, Crow R, Ambrose M, Alonso A: A clinical risk score for atrial fibrillation in a biracial prospective cohort (from the Atherosclerosis Risk in Communities [ARIC] study). Am J Cardiol 2011, 107:85-91.

32. Schnabel RB, Larson MG, Yamamoto JF, Sullivan LM, Pencina MJ, Meigs JB, Tofler GH, Selhub J, Jacques PF, Wolf PA, Magnani JW, Ellinor PT, Wang TJ, Levy D, Vasan RS, Benjamin EJ: Relations of biomarkers of distinct pathophysiological pathways and atrial fibrillation incidence in the community. Circulation 2010, 121:200-207.

33. Smith JG, Newton-Cheh C, Almgren P, Struck J, Morgenthaler NG, Bergmann A, Platonov PG, Hedblad B, Engstrom G, Wang TJ, Melander O: Assessment of conventional cardiovascular risk factors and multiple biomarkers for the prediction of incident heart failure and atrial fibrillation. J Am Coll Cardiol 2010, 56:1712-1719.

34. The Atherosclerosis Risk in Communities (ARIC) Study: design and objectives. The ARIC investigators.. Am J Epidemiol 1989, 129:687-702.

35. Fried $L P$, Borhani NO, Enright $P$, Furberg $C D$, Gardin $J M$, Kronmal RA, Kuller LH, Manolio TA, Mittelmark MB, Newman A, O'Leary DH, Psaty B, Rautaharju P, Tracy RP, Weiler PG: The Cardiovascular Health Study: design and rationale. Ann Epidemiol 1991, 1:263-276.

36. Feinleib M, Kannel WB, Garrison RJ, McNamara PM, Castelli WP: The Framingham Offspring Study. Design and preliminary data. Prev Med 1975, 4:518-525.

37. Pencina MJ, D'Agostino RB Sr, Steyerberg EW: Extensions of net reclassification improvement calculations to measure usefulness of new biomarkers. Stat Med 2011, 30:11-21.

38. Vienna: R Foundation for Statistical Computing. R Development Core Team: R: a language and environment for statistical computing. [http://www.Rproject.org], Version 2.8.0. 
39. Guey LT, Kravic J, Melander O, Burtt NP, Laramie JM, Lyssenko V, Jonsson A, Lindholm E, Tuomi T, Isomaa B, Nilsson P, Almgren P, Kathiresan S, Groop L, Seymour AB, Altshuler D, Voight BF: Power in the phenotypic extremes: a simulation study of power in discovery and replication of rare variants. Genet Epidemiol 2011, Feb 9. doi: 10.1002/gepi.20572.

40. Wolf PA, Mitchell JB, Baker CS, Kannel WB, D'Agostino RB: Impact of atrial fibrillation on mortality, stroke, and medical costs. Arch Intern Med 1998, 158:229-234.

41. Fox CS, Parise H, D'Agostino RB Sr, Lloyd-Jones DM, Vasan RS, Wang TJ, Levy D, Wolf PA, Benjamin EJ: Parental atrial fibrillation as a risk factor for atrial fibrillation in offspring. JAMA 2004, 291:2851-2855.

doi:10.1186/gm480

Cite this article as: Mihaescu et al.: Incremental value of rare genetic variants for the prediction of multifactorial diseases. Genome Medicine 2013 5:76.

\section{Submit your next manuscript to BioMed Central} and take full advantage of:

- Convenient online submission

- Thorough peer review

- No space constraints or color figure charges

- Immediate publication on acceptance

- Inclusion in PubMed, CAS, Scopus and Google Scholar

- Research which is freely available for redistribution

Submit your manuscript at www.biomedcentral.com/submit 